\title{
PLCB4 upregulation is associated with unfavorable prognosis in pediatric acute myeloid leukemia
}

\author{
SHIWEN WU ${ }^{1}$, WEI ZHANG ${ }^{2}$, DONGQIN SHEN ${ }^{3}$, JIANLE LU $^{2}$ and LI ZHAO ${ }^{2}$ \\ Departments of ${ }^{1}$ Clinical Laboratory, ${ }^{2}$ Central Laboratory and ${ }^{3}$ Nursing, \\ The First Hospital of Lanzhou University, Lanzhou University, Lanzhou, Gansu 730000, P.R. China
}

Received May 7, 2019; Accepted August 21, 2019

DOI: $10.3892 / \mathrm{ol} .2019 .10921$

\begin{abstract}
Phospholipase $\mathrm{C}(P L C)$ is a membrane-associated enzyme that regulates several cellular behaviors including cell motility, growth, transformation and differentiation. PLC is involved in cancer migration, invasion and drug resistance. However, the expression status and prognostic role of PLCB4 in acute myeloid leukemia (AML) remain unclear. In the present study, the complete clinical and mRNA expression data of 285 pediatric patients with de novo AML were obtained from the Therapeutically Available Research to Generate Effective Treatments database. The association between PLCB4 expression and clinical and molecular features was explored. The expression of $P L C B 4$ was significantly higher in patients with AML who relapsed compared with those with long-term complete remission. Patients with PLCB4 upregulation had significantly lower overall survival (OS) and event free survival (EFS) rate compared with those with low PLCB4 expression. Multivariate Cox's regression analyses demonstrated that high $P L C B 4$ expression was an independent risk factor of adverse OS $(\mathrm{P}<0.01 ; \mathrm{HR}, 2.081)$ and EFS $(\mathrm{P}<0.01 ; \mathrm{HR}, 2.130)$. Following stratification analysis according to transplant status in cases of first complete remission, the patients with high expression of PLCB4 had significantly lower OS and EFS rate in the chemotherapy group, but not the stem cell transplant group. Furthermore, PLCB4-associated genes were identified using Spearman's rank correlation analysis. KEGG pathway analysis revealed that $P L C B 4$ and its associated genes were mainly involved in three potential pathways, including the Rap1 signaling pathway. Overall, the findings of the present study suggest that increased PLCB4 expression is associated with poor clinical outcome in pediatric patients with AML, and thus may represent a potential prognostic biomarker and therapeutic target for AML.
\end{abstract}

Correspondence to: Dr Li Zhao, Department of Central Laboratory, The First Hospital of Lanzhou University, Lanzhou University, 1 Donggang West Road, Lanzhou, Gansu 730000, P.R. China E-mail: zhaoli@1zu.edu.cn

Key words: phospholipase C $\beta 4$, acute myeloid leukemia, prognosis, pediatric, recurrence

\section{Introduction}

Acute myeloid leukemia (AML) is a highly heterogeneous disease, characterized by poor differentiation and abnormal proliferation of myeloid progenitors (1). AML is the most common type of acute leukemia in adults, with an estimated annual incidence of $>20,000$ new cases in the United States in 2019 (2). In 2013 it was reported that it was the leading cause of cancer deaths among children and individuals $<35$ years of age in China (3). Although ongoing advances in the treatment of AML have led to significant improvements in the clinical outcome, the overall survival (OS) rate remains low, mainly due to high recurrence rate and drug resistance (4). The specific mechanisms involved in these processes remain poorly defined.

Phospholipase $\mathrm{C}(P L C)$ plays an essential role in cell metabolism, as it hydrolyzes membrane-bound phospholipid phosphatidylinositol 4,5-biphosphate (PIP2) into two second messengers, inositol 1,4,5-triphosphate (IP3) and diacylglycerol (DAG) (5). A growing body of evidence indicates that increased expression of $P L C$ promotes invasion and metastasis in several solid tumor types, including gastric carcinoma, breast cancer, hepatocellular carcinoma, pancreatic cancer, esophageal cancer and colorectal cancer $(6,7)$.

Of the $P L C$ isoforms, $P L C B 1$ promotes breast cancer cell migration via actin remodeling (8), and additionally regulates cell cycle progression in AML (9-12). PLCB2 is significantly upregulated in human breast cancer and exhibits oncogenic functions and poor prognostic effects, by promoting cell division, motility and invasion $(13,14)$.

$P L C B 4$ encodes the $\beta 4$ isoform of $P L C$ isoenzymes, a superfamily that regulates the metabolism of inositol lipids (15). Increased PLCB4 expression is associated with poor OS rate in patients with solid tumors, including mesothelioma, melanoma and gastrointestinal tumors (16-18). PLCB4 contributes to solid tumor progression $(16,17)$, however, its function in hematological tumors, particularly AML, has not been explored. Leukemia stem cells (LSC) are the source of drug resistance and relapse of AML, however, PLCB4 expression in LSCs remain to be elucidated (19). In the present study, the effect of $P L C B 4$ expression and its clinical significance in AML were investigated. PLCB4 expression may serve as a novel diagnostic or therapeutic target in pediatric patients with AML. 
A

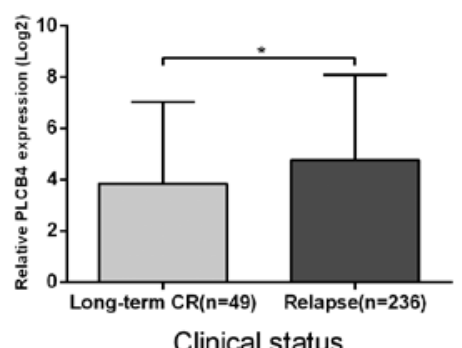

B

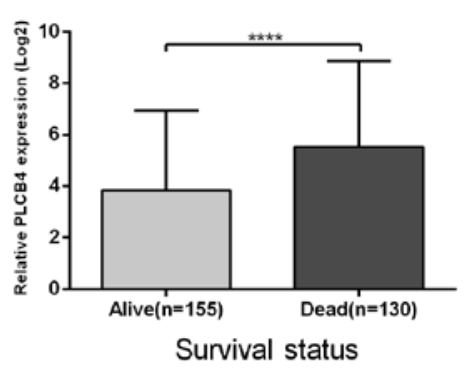

Figure 1. PLCB4 expression in AML. (A) PLCB4 expression in patients with AML of different clinical status (relapse and long-term CR). (B) PLCB4 expression in AML patients of different survival status (alive and deceased) at 5-years follow-up. ${ }^{*} \mathrm{P}<0.05 ;{ }^{* * * *} \mathrm{P}<0.0001$. AML, acute myeloid leukemia; CR, complete remission; $P L C B 4$, phospholipase C $\beta 4$.

\section{Materials and methods}

Patients. All patients and corresponding clinical information were obtained from the Therapeutically Available Research to Generate Effective Treatments (TARGET) database (https://ocg.cancer.gov/programs/target/) on 11 November 2018. Samples were excluded if clinical information and RNA expression data were incomplete. A total of 285 pediatric patients with de novo AML were enrolled (median age, 10 years; range, 0-23 years), including 147 males and 138 females. Of these patients, 36 accepted stem cell transplants (SCTs) in first complete remission (CR1), and the remaining patients accepted chemotherapy. Median follow-up time of the patients was 1,355 days (range, 2-4,037 days).

Gene expression analyses. The raw counts of mRNA expression profiles (level 3) were downloaded from the TARGET database and $\log _{2}$ transformed. Patients were divided into high- and low-PLCB4 groups, using the median of PLCB4 expression level as the cut-off value. A comparison of clinical and molecular characteristics was performed between patients with high and low PLCB4 expression.

Functional and pathway enrichment analysis of PLCB4-associated genes in AML. Spearman's rank correlation analysis was performed to identify $P L C B 4$-associated genes using R software (version 3.3.3; www.r-project.org). Correlation coefficient values $<-4$ or $>4$ were considered to be significantly correlated with $P L C B 4$ expression. Gene Ontology (GO; http://geneontology.org) functional analysis (20) and Kyoto Encyclopedia of Genes and Genomes (KEGG; http://david.ncifcrf.gov) pathway enrichment analysis $(21,22)$ of the PLCB4-associated genes were performed using the online Database for Annotation, Visualization and Integrated Discovery database (version 6.8; https://david. ncifcrf.gov/) (23). $\mathrm{P}<0.05$ was considered as the cut-off criterion for significant differences.

PLCB4 expression in leukemia stem cells. The expression of PLCB4 in primitive progenitor cells was assessed, based on the GSE30377 database (https://www.ncbi.nlm.nih. gov/geo/), in which gene expression data was obtained from 23 primary human AML samples and sorted into stem cells and progenitors, according to the expression of CD34 and CD38 markers (24).
Statistical analysis. Continuous variables were presented as the median with interquartile range, and categorical variables were presented as frequencies and percentage proportions. Pearson's $\chi^{2}$ or Fisher's exact tests were performed for categorical variables. Mann-Whitney U tests were performed to analyze the difference of continuous variables between two groups, while Kruskal-Wallis test were used for the comparison of multiple groups, and a Bonferroni test for the post hoc test. OS was defined as the time from diagnosis until death for any reason, or until the last follow-up. Event-free survival (EFS) was defined as the time from diagnosis to death, relapse, induction failure or last follow-up. Survival analysis was based on the Kaplan-Meier method and the log-rank tests to compare the differences between survival curves. Univariate and multivariate Cox's regression analyses were used to assess the association between $P L C B 4$ expression and prognosis, as well as other clinical variables. The sensitivity and specificity of the PLCB4 expression signature was evaluated according to the area under the curve (AUC) of receiver operating characteristic (ROC) curves of 5-year OS and EFS. ROC curves were generated using the survival ROC packages based on R software (version 3.3.3; www.r-project.org/). The SPSS software (version 23.0; IBM Corp.) was applied for statistical analysis. The figures were generated using GraphPad Prism (version 6.01; GraphPad Software, Inc.) or R software. $\mathrm{P}<0.05$ was considered to indicate a statistically significant difference.

\section{Results}

PLCB4 expression in AML. Amongst the 285 patients, 49 remained in long-term complete remission (CR) until the last follow-up, and 236 experienced relapse. PLCB4 expression was significantly upregulated in patients with relapse (median, 4.858) compared with patients with long-term CR (median, 4.087) $(\mathrm{P}<0.05$; Fig. 1A). Significant differences were also observed in the classification of survival status ( $<<0.01$; Fig. 1B). Median PLCB4 mRNA expression was significantly lower in patients who survived (median, 3.807) compared with those that had died (median, 5.473) at 5-years follow-up. No significant differences in PLCB4 expression were observed amongst karyotype and gender classifications ( $\mathrm{P}=0.263$ and 0.509 , respectively) (data not shown).

Association between PLCB4 expression and clinical characteristics. The clinical and molecular features of patients 
Table I. Comparison of clinical and molecular characteristics with PLCB4 expression in patients with acute myeloid leukemia.

\begin{tabular}{|c|c|c|c|}
\hline Characteristic & Low PLCB4 $(\mathrm{n}=142)$ & High PLCB4 $(\mathrm{n}=143)$ & P-value \\
\hline Age, median (range) years & $11(0-22)$ & $10(0-23)$ & 0.493 \\
\hline Sex, n $(\%)$ & & & 0.259 \\
\hline Male & $78(54.9)$ & $69(48.3)$ & \\
\hline Female & $64(45.1)$ & $74(51.7)$ & \\
\hline Race, n (\%) & & & 0.510 \\
\hline Caucasian & $108(76.1)$ & $102(71.3)$ & \\
\hline African American & $13(9.2)$ & $19(13.3)$ & \\
\hline Asian & $6(4.2)$ & $3(2.1)$ & \\
\hline Other & $7 \quad(4.9)$ & $6 \quad(4.2)$ & \\
\hline Unknown & $8 \quad(5.6)$ & $13(9.1)$ & \\
\hline WBC, median (range) $\times 10^{9} / 1$ & $59.8(0.9-446)$ & $28.6(2-519)$ & $<0.01$ \\
\hline BM blast, median (range), $\%$ & $73(20-100)$ & $73(14-99)$ & 0.473 \\
\hline PB blast, median (range), $\%$ & $63(0-97)$ & $59(0-97)$ & 0.012 \\
\hline FAB subtypes, n (\%) & & & $<0.01$ \\
\hline M0 & $2(1.4)$ & $5 \quad(3.5)$ & \\
\hline M1 & $16(11.3)$ & $21(14.7)$ & \\
\hline M2 & $29(20.4)$ & $41(28.7)$ & \\
\hline M4 & $52(36.6)$ & $13(9.1)$ & \\
\hline M5 & $22(15.5)$ & $32(22.4)$ & \\
\hline M6 & $2(1.4)$ & $2(1.4)$ & \\
\hline M7 & $1(0.7)$ & $8 \quad(5.6)$ & \\
\hline NOS & $9(6.3)$ & $8 \quad(5.6)$ & \\
\hline Unknown & $9(6.3)$ & $13 \quad(9.1)$ & \\
\hline Karyotype, n (\%) & & & 0.536 \\
\hline Normal & $33(23.2)$ & $38(26.6)$ & \\
\hline Abnormal & $100(67.8)$ & $97(67.8)$ & \\
\hline Unknown & $9(6.3)$ & $8(5.6)$ & \\
\hline SCT in 1 st CR, n (\%) & & & 0.400 \\
\hline Yes & $16(11.3)$ & $20(14.0)$ & \\
\hline No & $117(82.4)$ & $108(75.5)$ & \\
\hline CR status at end of course $1, \mathrm{n}(\%)$ & & & 0.064 \\
\hline Yes & $116(81.7)$ & $104(72.7)$ & \\
\hline No & $24(16.9)$ & $37(25.9)$ & \\
\hline CR status at end of course $2, \mathrm{n}(\%)$ & & & 0.298 \\
\hline Yes & $122(85.9)$ & $120(83.9)$ & \\
\hline No & $13(9.2)$ & $19(13.3)$ & \\
\hline MRD at end of course $1, \mathrm{n}(\%)$ & & & 0.011 \\
\hline Yes & $28(19.7)$ & $46(32.2)$ & \\
\hline No & $76(53.5)$ & $59(41.3)$ & \\
\hline MRD at end of course $2, \mathrm{n}(\%)$ & & & 0.210 \\
\hline Yes & $17(12.0)$ & $23(16.1)$ & \\
\hline No & $81(57.0)$ & $70(49.0)$ & \\
\hline Induction failure, n (\%) & $11(7.7)$ & $17(11.9)$ & 0.240 \\
\hline Relapse, n (\%) & $110(77.5)$ & $126(88.1)$ & 0.017 \\
\hline
\end{tabular}

PLCB4, phospholipase C $\beta 4$; WBC, white blood cells; BM, bone marrow; PB, peripheral blood; FAB, French-American-British classification; $\mathrm{SCT}$, stem cell transplantation; $\mathrm{CR}$, complete remission; MRD, minimal residual disease.

were compared between high- and low- $P L C B 4$ groups, in order to determine the association of $P L C B 4$ expression with
AML (Table I). Patients with low PLCB4 expression had higher white blood cell counts (median, 59.8) vs. patients with 
high PLCB4 expression (median, 28.6) ( $\mathrm{P}<0.01)$. Significant differences were found in both FAB subtypes $(\mathrm{P}<0.01)$ and peripheral blood $(\mathrm{PB})$ blast $(\mathrm{P}=0.012)$ between the two groups. There were no significant associations between PLCB4 expression and race, gender, karyotype status, and PB and bone marrow blast percentages. Patients with high PLCB4 expression had a significantly higher relapse rate than those with low PLCB4 expression (88.1 vs. $77.5 \%$; $\mathrm{P}=0.017$ ). In addition, patients with high $P L C B 4$ expression had a higher incidence of minute residual disease (MRD) at the end of the first course of chemotherapy compared with those with low PLCB4 expression (32.2 vs. $19.7 \% ; \mathrm{P}=0.011)$. No significant differences were observed at the end of the second course of chemotherapy $(\mathrm{P}=0.210)$ between patients with low and high PLCB4 expression. Patients with high PLCB4 expression had a tendency to have lower $\mathrm{CR}$ rates at the end of the first course (72.7 vs. 81.7\%; $\mathrm{P}=0.064)$ and second course of therapy ( 83.9 vs. $85.9 \% ; \mathrm{P}=0.298$ ) compared with those with low PLCB4 expression; however, the differences were not statistically significant.

Association between PLCB4 expression and genetic mutations. Additionally, the association between PLCB4 expression and the molecular characteristics of patients was investigated. No significant differences were detected in the mutation frequencies of Fms-related tyrosine kinase 3 (FLT3) internal tandem duplication (ITD) or point mutation, nucleophosmin 1 (NPM1), CCAAT enhancer binding protein $\alpha$ (CEBPA) and Wilms' tumor gene 1 (WT1) between the two PCLB4 expression groups (Table II).

Association of PLCB4 expression and prognosis. To determine the prognostic value of PLCB4 expression in AML, Kaplan-Meier curves were generated to examine the association between PLCB 4 expression and patient survival. Patients with high $P L C B 4$ expression had shorter OS (median, 28.5 vs. 60.7 months) and EFS (median, 12.5 vs. 16.3 months) time (Fig. 2A and B). The prognostic value of PLCB4 expression was further confirmed using Cox's regression analyses (univariate and multivariate). As presented in Table III, univariate analysis indicated that PLCB4 overexpression (HR, 1.905; P<0.01), FLT3-ITD-positive (HR, 1.681; $\mathrm{P}=0.017$ ), and $W T 1$-mutated (HR, 1.827; $\mathrm{P}=0.029)$ were associated with shorter OS time, while the mutations in NPM1 (HR, 0.451; $\mathrm{P}=0.081$ ) and $C E B P A(\mathrm{HR}, 0.215 ; \mathrm{P}=0.031$ ) were favorable for OS time. Furthermore, patients with $P L C B 4$ overexpression (HR, 1.903; $\mathrm{P}<0.01$ ), as well as FLT3-ITD-positive (HR, 1.634; $\mathrm{P}=0.023$ ), and WT1-mutated (HR, 1.988; $\mathrm{P}=0.013$ ) genotypes had shorter EFS time. NPM1-mutated (HR, 0.403; $\mathrm{P}=0.046)$ and $C E B P A$-mutated (HR, 0.185; $\mathrm{P}=0.018$ ) genotypes were associated with longer EFS time. Multivariate analysis revealed high PLCB4 expression was an independent prognostic factor for shorter OS time $(\mathrm{P}<0.01 ; \mathrm{HR}, 2.081)$ and $\mathrm{EFS}(\mathrm{P}<0.01$; HR, 2.130) in AML. Notably, when patients were stratified according to transplant status in CR1, in the chemotherapy group, patients with high PLCB4 expression had significantly shorter OS $(\mathrm{P}<0.01)$ and EFS $(\mathrm{P}<0.01)$ times compared with those with low $P L C B 4$ expression (Fig. $2 \mathrm{C}$ and D). The results were unaffected by multivariate adjustments for clinical and genetic mutation variables: $\mathrm{OS}(\mathrm{P}<0.01 ; \mathrm{HR}, 2.239)$ and
Table II. Comparison of genetic mutations and PLCB4 expression in patients with acute myeloid leukemia.

\begin{tabular}{lrrr}
\hline Gene mutation & $\begin{array}{c}\text { Low PLCB4 } \\
(\mathrm{n}=142)\end{array}$ & $\begin{array}{c}\text { High PLCB4 } \\
(\mathrm{n}=143)\end{array}$ & P-value \\
\hline $\begin{array}{l}\text { FLT3-ITD, n (\%) } \\
\text { Yes }\end{array}$ & $26(18.3)$ & $21(14.7)$ & 0.156 \\
$\quad$ No & $116(81.7)$ & $122(85.3)$ & \\
$\begin{array}{l}\text { FLT3-PM, n (\%) } \\
\text { Yes }\end{array}$ & $13(9.2)$ & $7(4.9)$ & 0.159 \\
No & $128(90.1)$ & $135(94.4)$ & \\
NPM1,n (\%) & & & 0.268 \\
Yes & $7(4.9)$ & $12(8.4)$ & \\
No & $128(90.1)$ & $128(89.5)$ & \\
CEBPA, n (\%) & $9(6.3)$ & $7(4.9)$ & 0.596 \\
Yes & $131(92.3)$ & $134(93.7)$ & \\
No & & & 0.052 \\
WT1,n $(\%)$ & $17(12.0)$ & $8(5.6)$ & \\
Yes & $120(84.5)$ & $132(92.3)$ & \\
No &
\end{tabular}

PLCB4, phospholipase C $\beta 4 ;$ FLT3-ITD, internal tandem duplication of the FLT3 gene; FLT3-PM, FLT3 point mutation at codon 835-836; $N P M 1$, nucleophosmin 1; CEBPA, CCAAT-enhancer binding protein $\alpha ; W T 1$, Wilms tumor gene 1 .

EFS $(\mathrm{P}<0.01 ; \mathrm{HR}, 2.311)$ times. No significant differences between high and low PLCB4 expression groups of patients undergoing $\mathrm{SCT}$ were observed (OS, $\mathrm{P}=0.812$; $\mathrm{EFS}, \mathrm{P}=0.833$ ) (Fig. 2E and F). Overall, these data suggest PLCB4 overexpression may be an independent predictor of poor prognosis in patients receiving chemotherapy, but not undergoing $\mathrm{SCT}$ in CR1.

Discriminative capacity of PLCB4 expression. In order to evaluate the clinical utility of $P L C B 4$ expression as a prognostic biomarker of AML, the AUC of ROC curves were used to determine the discriminative capacity of $P L C B 4$ expression to predict 5-year survival rates. The AUC values were high for the 5-year ROC curves of OS and EFS (AUC, 0.654 and 0.657, respectively; Fig. 3A and B), and similar results were observed for OS and EFS times of patients treated with chemotherapy in CR1 (AUC, 0.649 and 0.65, respectively; Fig. 3C and D). Overall, these findings suggest that $P L C B 4$ expression may serve as a potential prognostic biomarker of AML.

Functional and pathway enrichment analysis of PLCB4-associated genes in AML. In order to obtain insights into the biological functions and potential mechanisms of $P L C B 4$ in AML, $P L C B 4$-associated genes were identified using Spearman's rank correlation analysis. A total of 648 mRNAs were significantly correlated with PLCB4 expression. Of these, 14 genes were negatively correlated and 634 genes were positively correlated. PCYTIB, PARD6B, RAB3IP, CALDI and $A L D H 1 A 1$ were the top 5 genes that positively correlated with the expression levels of PLCB4 according to the value 

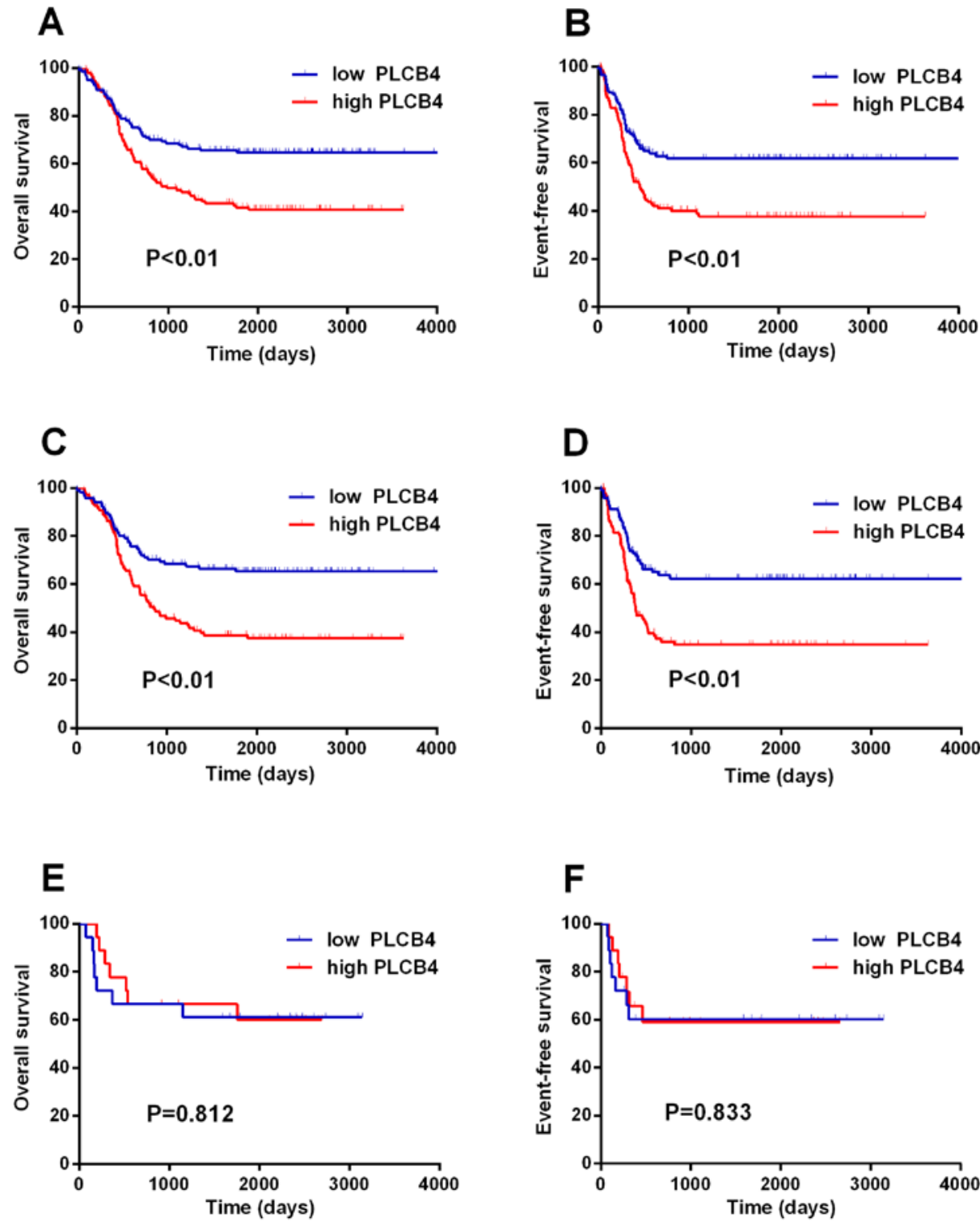

Figure 2. Survival analysis of patients with AML according to PLCB4 gene expression. (A and B) Patients with high PLCB4 expression had significantly shorter OS and EFS times compared with patients with low expression. (C and D) Patients that were treated with chemotherapy in CR1 and had high PLCB4 expression had significantly shorter OS and EFS times compared with patients with low expression. (E and F) No significant differences were observed in OS and EFS times between high- and low-PLCB4 expression groups in patients undergoing SCT in CR1. PLCB4, phospholipase C $\beta 4$; SCT, stem cell transplants; OS, overall survival; EFS, event-free survival; CR1, first complete remission.

of the correlation coefficient. Subsequently, GO functional and KEGG pathway enrichment analysis were conducted, based on the genes that were correlated with PLCB4 expression. The correlated genes were significantly enriched in the pathways associated with 'regulation of transcription', ' $\mathrm{G}_{2} / \mathrm{M}$ transition of mitotic cell cycle', 'centriole replication', 'cilium assembly' and 'calcium-dependent cell-cell adhesion via plasma membrane cell adhesion molecules' (Fig. 4). KEGG pathway analysis predicted three potential pathways that were associated with $P L C B 4$ and its correlated genes and were regulated during $A M L$, including the thyroid hormone signaling pathway, RAP1 signaling and platelet activation (Table IV).

mRNA expression of PLCB4 in leukemia stem cells. Amongst all cell populations that were assessed, the expression of $P L C B 4$ was highest in $\mathrm{CD} 34^{+} \mathrm{CD} 38$ - cells compared with both
CD34 $/ \mathrm{CD}^{-} 8^{+}$and $\mathrm{CD} 34^{+} \mathrm{CD} 38^{-}$cells $(\mathrm{P}=0.020$ and 0.029 , respectively; Fig. 5).

\section{Discussion}

Despite improvements in the prognosis of AML, several clinical challenges remain for this disease. High relapse rates remain the major cause of treatment failure in patients with AML and CR1, who are treated with intensive chemotherapy alone (1). The present study demonstrated that increased $P L C B 4$ expression was associated with a high risk of relapse and death, whereas low expression of PLCB4 was associated with favorable prognosis in patients with AML. ROC curve and Cox's regression analyses for OS and EFS time of patients with AML further confirmed that PLCB4 expression was considered as an independent prognostic indicator. Furthermore, 
Table III. Univariate and multivariate analyses of prognostic factors for OS and EFS in AML patients.

A, Univariate analysis

\begin{tabular}{|c|c|c|c|c|}
\hline \multirow[b]{2}{*}{ Variables } & \multicolumn{2}{|c|}{ OS } & \multicolumn{2}{|c|}{ EFS } \\
\hline & HR (95\% CI) & P-value & HR $(95 \%$ CI $)$ & P-value \\
\hline PLCB4 & $1.905(1.335-2.718)$ & $<0.01$ & $1.903(1.334-2.714)$ & $<0.01$ \\
\hline Age & $1.022(0.992-1.054)$ & 0.156 & $1.014(0.983-1.045)$ & 0.382 \\
\hline Sex & $1.308(0.927-1.845)$ & 0.127 & $1.288(0.913-1.817)$ & 0.150 \\
\hline Race & $1.005(0.797-1.268)$ & 0.963 & $0.958(0.759-1.209)$ & 0.718 \\
\hline FAB & $1.071(0.981-1.169)$ & 0.127 & $1.091(0.998-1.191)$ & 0.055 \\
\hline WBC & $1.000(0.998-1.002)$ & 0.894 & $1.000(0.998-1.002)$ & 0.841 \\
\hline BM blast & $0.998(0.990-1.007)$ & 0.703 & $0.997(0.989-1.006)$ & 0.512 \\
\hline PB blast & $0.996(0.990-1.002)$ & 0.206 & $0.996(0.990-1.002)$ & 0.175 \\
\hline Karyotype & $1.103(0.737-1.651)$ & 0.634 & $1.110(0.741-1.662)$ & 0.612 \\
\hline SCT in 1 st CR & $0.842(0.482-1.471)$ & 0.545 & $0.804(0.460-1.404)$ & 0.442 \\
\hline FLT3-ITD & $1.681(1.099-2.571)$ & 0.017 & $1.634(1.069-2.497)$ & 0.023 \\
\hline FLT3-PM & $0.474(0.194-1.158)$ & 0.101 & $0.445(0.182-1.088)$ & 0.076 \\
\hline NPM1 & $0.451(0.184-1.103)$ & 0.081 & $0.403(0.164-0.986)$ & 0.046 \\
\hline CEBPA & $0.215(0.053-0.868)$ & 0.031 & $0.185(0.046-0.748)$ & 0.018 \\
\hline$W T 1$ & $1.827(1.065-3.134)$ & 0.029 & $1.988(1.158-3.412)$ & 0.013 \\
\hline
\end{tabular}

B, Multivariate analyses

\begin{tabular}{lcccc}
\hline & \multicolumn{2}{c}{ OS } & & EFS \\
\cline { 2 - 3 } Variables & HR $(95 \% \mathrm{CI})$ & P-value & HR $(95 \%$ CI $)$ & P-value \\
\hline PLCB4 & $2.081(1.440-3.008)$ & $<0.01$ & $2.130(1.447-3.137)$ & $<0.01$ \\
FAB & - & - & $1.099(1.004-1.203)$ & 0.040 \\
FLT3-ITD & $1.709(1.066-2.742)$ & 0.026 & $0.526(0.192-1.440)$ & 0.052 \\
FLT3-PM & - & - & $0.340(0.132-0.875)$ & 0.025 \\
NPM1 & $0.317(0.126-0.798)$ & 0.015 & $0.194(0.048-0.791)$ & 0.022 \\
CEBPA & $0.213(0.053-0.864)$ & 0.030 & $1.807(0.964-3.386)$ & 0.065 \\
WT1 & $1.536(0.835-2.825)$ & 0.167 & $0.902)$ & \\
\hline
\end{tabular}

Multivariate analysis included variables with $\mathrm{P}<0.1$ in univariate analysis of OS and EFS. HR $\geq 1.0$ indicated a higher risk for OS and EFS, whilst HR $\leq 1.0$ indicated a lower risk. PLCB4, phospholipase C $\beta 4$; CI, confidence interval; HR, hazard ratio; EFS, event-free survival; OS, overall survival; WBC, white blood cell; BM, bone marrow; PB, peripheral blood; FAB, French-American-British classification; SCT, stem cell transplantation; CR, complete remission; FLT3-ITD, internal tandem duplication of the FLT3 gene; FLT3-PM, FLT3 point mutation at codon 835-836; NPM1, nucleophosmin; CEBPA, CCAAT-enhancer binding protein $\alpha$; WT1, Wilms tumor gene 1.

Table IV. Kyoto Encyclopedia of Genes and Genomes pathway analysis prediction of potential pathways in which $P L C B 4$ and $P L C B 4$-associated genes were enriched in acute myeloid leukemia.

\begin{tabular}{|c|c|c|}
\hline Pathway ID & Pathway name & Genes \\
\hline hsa04919 & Thyroid hormone signaling pathway & $\begin{array}{l}\text { SLC16A2, HDAC2, PLCB4, THRB, SLCO1C1, TBC1D4, ITGB3, } \\
\text { PIK3R3, MED12L }\end{array}$ \\
\hline hsa04015 & RAP1 signaling pathway & $\begin{array}{l}\text { PARD6B, IGF1R, MAGI3, PLCB4, TIAM1, TEK, RAPGEF6, } \\
\text { RAPGEF5, ITGB3, RAPGEF2, EGF }, \text { PIK } 3 R 3\end{array}$ \\
\hline hsa04611 & Platelet activation & $\begin{array}{l}\text { PLCB4, PPP1R12A, COL2A1, PRKG2, ITGB3, PIK3R3, COL11A1, } \\
\text { COL5A1 }\end{array}$ \\
\hline
\end{tabular}


A

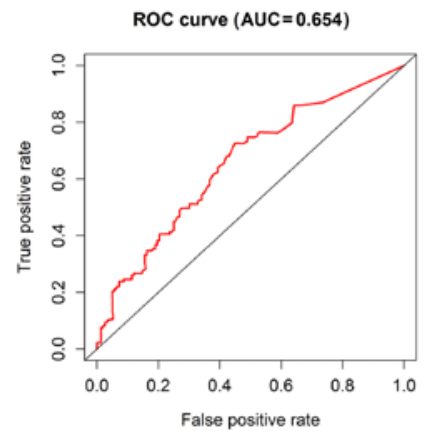

C

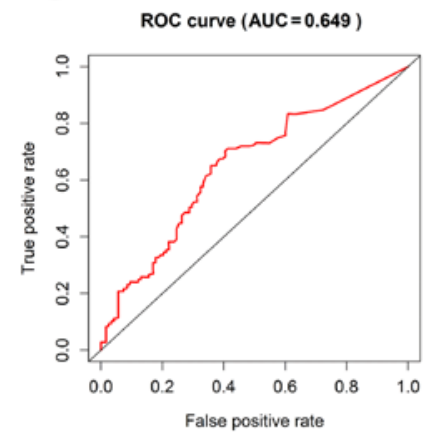

B

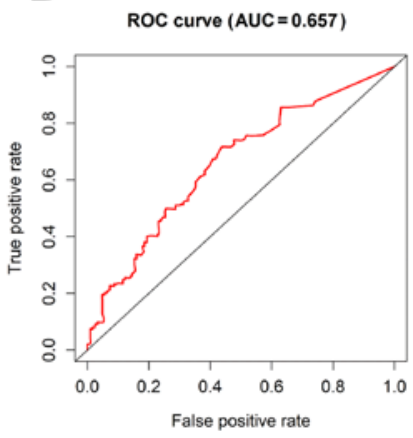

D

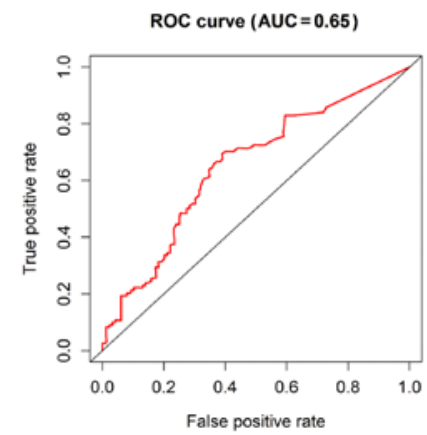

Figure 3. ROC analysis of phospholipase C $\beta 4$ expression to predict 5-year OS and EFS. ROC predicts 5-year (A) OS and (B) EFS of all patients in the cohort, and (C) OS and (D) EFS of patients treated with chemotherapy. ROC, receiver operating characteristics; OS, overall survival; EFS, event-free survival; AUC, area under the ROC curve.

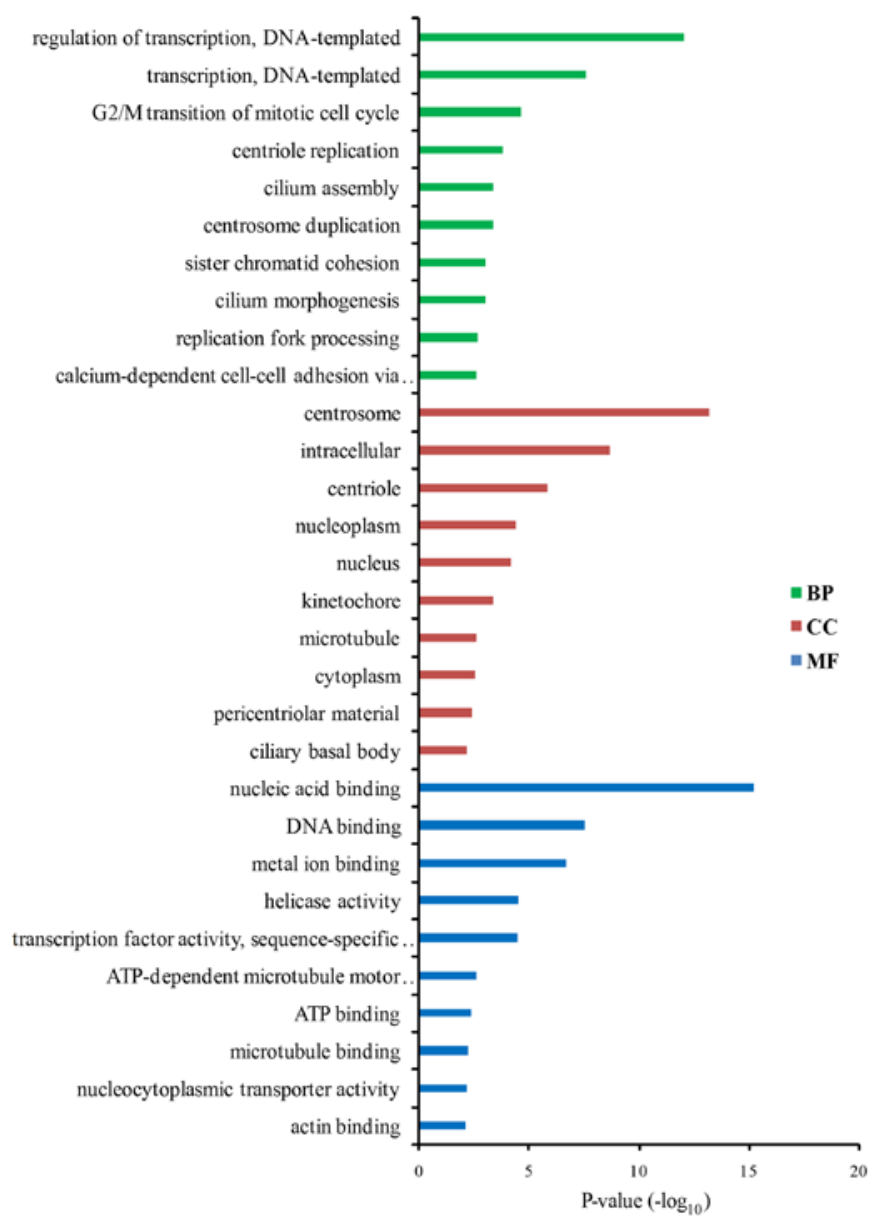

Figure 4. Functional enrichment analysis of PLCB4 and associated genes in AML. GO analysis of PLCB4 and associated genes using the Database for Annotation, Visualization and Integrated Discovery database in AML showed $143 \mathrm{GO}$ terms enriched with these genes. The top 10 enriched GO terms were classified into CC, MF and BP. PLCB4; phospholipase C $\beta 4$; GO, Gene Ontology; BP, biological processes; CC, cell components; MF, molecular functions; AML, acute myeloid leukemia. 


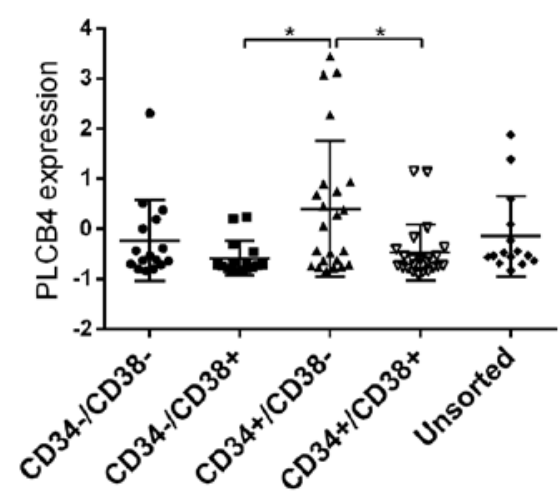

Figure 5. $P L C B 4$ mRNA expression in leukemia stem cell populations Comparison of $P L C B 4$ mRNA expression differences among the following sorted cell populations: $\mathrm{CD} 34^{+} \mathrm{CD} 38^{+}, \mathrm{CD} 34^{+} \mathrm{CD} 38^{-}, \mathrm{CD} 34^{-} \mathrm{CD} 38^{+}$, CD34 ${ }^{-}$D $38^{-}$and unsorted cell populations according to the $\mathrm{CD} 34$ and $\mathrm{CD} 38$ markers of leukemia stem cells. " $\mathrm{P}<0.05$. PLCB4; phospholipase $\mathrm{C} \beta 4$.

high $P L C B 4$ expression was associated with an unfavorable outcome in patients with AML who received chemotherapy. Thus, PLCB4 represents a predictive molecular marker for the effectiveness of chemotherapy. However, further verification is required in larger cohorts.

High PLCB4 expression was reported in numerous cancer types and was associated with worse clinical outcomes for gastrointestinal tumors and mesothelioma, as well as melanomas $(17,18)$. The present study suggests that PLCB4 expression plays a vital role in tumor development and recurrence in patients with $A M L$, however the underlying mechanisms of PLCB4 in AML remain poorly understood. A previous study reported that $P L C B 4$ was upregulated in multidrug-resistant HL-60 cell lines compared with wild-type HL-60 cells (25), indicating its association with drug-resistance in leukemia.

The presence of MRD following induction and/or consolidation chemotherapy has been demonstrated to be a significant risk factor and predictive marker of relapse in patients with AML (5,26-29). A growing body of evidence suggests that MRD prior to hematopoietic cell transplantation is associated with adverse clinical prognosis in AML in CR1 $(30,31)$. Notably, this study's findings indicated that a positive effect of $P L C B 4$ overexpression on the incidence of MRD was observed in patients with AML and CR1, demonstrating that PLCB4 expression plays a role in the relapse of AML.

$\mathrm{CD} 34^{+} \mathrm{CD} 38^{-}$leukemia stem cells are resistant to chemotherapy, immune-evasive, and are associated with a lower $\mathrm{CR}$ rate following induction and an unfavorable prognosis in AML $(32,33)$. In the present study, PLCB4 was found to be highly expressed in $\mathrm{CD} 34^{+} \mathrm{CD} 38^{-}$populations and was significantly associated with ALDH1A1, an important marker of cancer stem cells (34). However, the specific mechanism of $P L C B 4$ in leukemia stem cells remain undefined.

To further clarify the impact of PLCB4 expression on the response to treatment and clinical outcomes in patients with AML, the genes that were significantly correlated with $P L C B 4$ expression were identified in the current study. GO and KEGG analysis were performed to examine the potential functional pathways of $P L C B 4$-associated genes involved in AML. RAP1 signaling regulates several biological processes, including cell polarity, proliferation, differentiation, adhesion and movement (35). Moreover, RAP signaling plays an essential role in the invasion and migration of leukemia cells through interaction with downstream target molecules (36). RAP guanine nucleotide exchange factors (RAPGEFs) act as a molecular switch by promoting the exchange of RAP1 from a GDP-bound state to the active GTP-bound state $(37,38)$. Notably, RAPGEF6, RAPGEF5 and RAPGEF2 were significantly correlated with PLCB4 expression. GO and KEGG analysis revealed $P L C B 4$ and PLCB4-associated genes were involved in 'regulation of transcription, DNA-templated', 'transcription, DNA-templated', ' $\mathrm{G}_{2} / \mathrm{M}$ transition of mitotic cell cycle', 'centriole replication' and 'RAP1 signaling pathway'. Thus, RAP1 signaling may be involved in AML cell migration and invasion, via activation of PLCB4. Further studies to confirm this hypothesis experimentally are required.

To the best of our knowledge, the present study is the first to evaluate the prognostic value of PLCB4 expression in AML. However, some potential limitations remain. The present study was based on information obtained from the TARGET database, which restricted the data available. Experiments on cell and animal models are required to understand and validate the role of PLCB4 expression in AML. Despite these limitations, the present study identified a direct association between PLCB4 expression and prognosis based on a large and representative population. Further studies are required to elucidate the potential molecular mechanisms of PLCB4 in AML.

In conclusion, upregulation of $P L C B 4$ was associated with a poor clinical outcome in patients with AML. PLCB4 may therefore be a potential prognostic biomarker and therapeutic target of AML.

\section{Acknowledgements}

Not applicable.

\section{Funding}

No funding was received.

\section{Availability of data and materials}

The datasets used and/or analyzed during the current study are available from the corresponding author on reasonable request.

\section{Authors' contributions}

LZ, SW and WZ contributed to the study design. DS contributed to downloading and processing the data. SW and JL analyzed the data and wrote the manuscript. All authors read and approved the final manuscript.

\section{Ethics approval and consent to participate}

Not applicable.

\section{Patient consent for publication}

Not applicable. 


\section{Competing interests}

The authors declare that they have no competing interests.

\section{References}

1. Döhner H, Weisdorf DJ and Bloomfield CD: Acute myeloid leukemia. N Engl J Med 373: 1136-1152, 2015.

2. Siegel RL, Miller KD and Jemal A: Cancer statistics, 2019. CA Cancer J Clin 69: 7-34, 2019.

3. Wang N, Feng YJ, Wang BH, Fang LW, Cong S, Li YC, Yin P, Zhou MG and Wang LH: Disease burden of leukemia in the Chinese population, in 1990 and 2013. Zhonghua Liu Xing Bing Xue Za Zhi 37: 783-787, 2016 (In Chinese).

4. Dohner H, Estey E, Grimwade D, Amadori S, Appelbaum FR, Büchner T, Dombret H, Ebert BL, Fenaux P, Larson RA, et al: Diagnosis and management of AML in adults: 2017 ELN recommendations from an international expert panel. Blood 129 : 424-447, 2017.

5. Cocco L, Follo MY, Manzoli L and Suh PG: Phosphoinositidespecific phospholipase $\mathrm{C}$ in health and disease. J Lipid Res 56 : $1853-1860,2015$

6. Follo MY, Manzoli L, Poli A, McCubrey JA and Cocco L: PLC and PI3K/Akt $/ \mathrm{mTOR}$ signalling in disease and cancer. Adv Biol Regul 57: 10-16, 2015.

7. Cheng M, Bhujwalla ZM and Glunde K: Targeting phospholipid metabolism in cancer. Front Oncol 6: 266, 2016.

8. Sengelaub CA, Navrazhina K, Ross JB, Halberg N and Tavazoie SF: PTPRN2 and PLC $\beta 1$ promote metastatic breast cancer cell migration through $\mathrm{PI}(4,5) \mathrm{P} 2$-dependent actin remodeling. EMBO J 35: 62-76, 2016.

9. Lo Vasco VR, Calabrese G, Manzoli L, Palka G, Spadano A, Morizio E, Guanciali-Franchi P, Fantasia D and Cocco L: Inositide-specific phospholipase $\mathrm{c}$ betal gene deletion in the progression of myelodysplastic syndrome to acute myeloid leukemia. Leukemia 18: 1122-1126, 2004.

10. Cocco L, Manzoli L, Palka G and Martelli AM: Nuclear phospholipase $C$ beta1, regulation of the cell cycle and progression of acute myeloid leukemia. Adv Enzyme Regul 45: 126-135, 2005.

11. Bavelloni A, Poli A, Fiume R, Blalock W, Matteucci A Ramazzotti G, McCubrey JA, Cocco L and Faenza I: PLC-beta 1 regulates the expression of miR-210 during mithramycin-mediated erythroid differentiation in K562 cells. Oncotarget 5: 4222-4231, 2014.

12. Bavelloni A, Dmitrienko GI, Goodfellow VJ, Ghavami A, Piazzi M, Blalock W, Chiarini F, Cocco L and Faenza I: PLC $\beta 1$ a and PLC $\beta 1 \mathrm{~b}$ selective regulation and cyclin D3 modulation reduced by kinamycin $\mathrm{F}$ during $\mathrm{k} 562$ cell differentiation. J Cell Physiol 230: 587-594, 2015.

13. Bertagnolo V, Benedusi M, Querzoli P, Pedriali M, Magri E, Brugnoli $\mathrm{F}$ and Capitani S: PLC-beta2 is highly expressed in breast cancer and is associated with a poor outcome: A study on tissue microarrays. Int J Oncol 28: 863-872, 2006.

14. Bertagnolo V, Benedusi M, Brugnoli F, Lanuti P, Marchisio M, Querzoli P and Capitani S: Phospholipase C-beta 2 promotes mitosis and migration of human breast cancer-derived cells. Carcinogenesis 28: 1638-1645, 2007.

15. Johansson P, Aoude LG, Wadt K, Glasson WJ, Warrier SK, Hewitt AW, Kiilgaard JF, Heegaard S, Isaacs T, Franchina M, et al Deep sequencing of uveal melanoma identifies a recurrent mutation in PLCB4. Oncotarget 7: 4624-4631, 2016.

16. Li CF, Liu TT, Chuang IC, Chen YY, Fang FM, Chan TC, Li WS and Huang HY: PLCB4 copy gain and PLCB4 overexpression in primary gastrointestinal stromal tumors: Integrative characterization of a lipid-catabolizing enzyme associated with worse disease-free survival. Oncotarget 8: 19997-20010, 2017.

17. Kakiuchi T, Takahara T, Kasugai Y, Arita K, Yoshida N, Karube K, Suguro M, Matsuo K, Nakanishi H, Kiyono T, et al: Modeling mesothelioma utilizing human mesothelial cells reveals involvement of phospholipase-C beta 4 in YAP-active mesothelioma cell proliferation. Carcinogenesis, Aug 24, 2016 (Epub ahead of print).

18. van de Nes JAP, Koelsche C, Gessi M, Möller I, Sucker A, Scolyer RA, Buckland ME, Pietsch T, Murali R, Schadendorf D, et al: Activating CYSLTR2 and PLCB4 mutations in primary leptomeningeal melanocytic tumors. J Invest Dermatol 137: 2033-2035, 2017.
19. Thomas D and Majeti R: Biology and relevance of human acute myeloid leukemia stem cells. Blood 129: 1577-1585, 2017.

20. Ashburner M, Ball CA, Blake JA, Botstein D, Butler H, Cherry JM, Davis AP, Dolinski K, Dwight SS, Eppig JT, et al: Gene ontology: Tool for the unification of biology. The gene ontology consortium. Nat Genet 25: 25-29, 2000.

21. Tanabe M and Kanehisa M: Using the KEGG database resource. Curr Protoc Bioinformatics, Chapter 1: Unit1.12, 2012.

22. Aoki KF and Kanehisa M: Using the KEGG database resource. Curr Protoc Bioinformatics, Chapter 1: Unit 1.12, 2005.

23. Huang da W, Sherman BT and Lempicki RA: Systematic and integrative analysis of large gene lists using DAVID bioinformatics resources. Nat Protoc 4: 44-57, 2009.

24. Eppert K, Takenaka K, Lechman ER, Waldron L, Nilsson B, van Galen P, Metzeler KH, Poeppl A, Ling V, Beyene J, et al: Stem cell gene expression programs influence clinical outcome in human leukemia. Nat Med 17: 1086-1093, 2011.

25. Zheng GH,Fu JR, Xu YH, Jin XQ, Liu WL and Zhou JF: Screening and cloning of multi-drug resistant genes in HL-60/MDR cells. Leuk Res 33: 1120-1123, 2009.

26. Ivey A, Hills RK, Simpson MA, Jovanovic JV, Gilkes A, Grech A, Patel Y, Bhudia N, Farah H, Mason J, et al: Assessment of minimal residual disease in standard-Risk AML. N Engl J Med 374: 422-433, 2016.

27. Terwijn M, van Putten WL, Kelder A, van der Velden VH, Brooimans RA, Pabst T, Maertens J, Boeckx N, de Greef GE, Valk PJ, et al: High prognostic impact of flow cytometric minimal residual disease detection in acute myeloid leukemia: Data from the HOVON/SAKK AML 42A study. J Clin Oncol 31: 3889-3897, 2013.

28. Chen X, Xie H, Wood BL, Walter RB, Pagel JM, Becker PS, Sandhu VK, Abkowitz JL, Appelbaum FR and Estey EH: Relation of clinical response and minimal residual disease and their prognostic impact on outcome in acute myeloid leukemia. J Clin Oncol 33: 1258-1264, 2015.

29. Schuurhuis GJ and Ossenkoppele G: Minimal residual disease in acute myeloid leukemia: Already predicting a safe haven? Expert Rev Hematol 3: 1-5, 2010.

30. Walter RB, Buckley SA, Pagel JM, Wood BL, Storer BE, Sandmaier BM, Fang M, Gyurkocza B, Delaney C, Radich JP, et al: Significance of minimal residual disease before myeloablative allogeneic hematopoietic cell transplantation for AML in first and second complete remission. Blood 122: 1813-1821, 2013.

31. Buckley SA, Wood BL, Othus M, Hourigan CS, Ustun C, Linden MA, DeFor TE, Malagola M, Anthias C, Valkova V, et al: Minimal residual disease prior to allogeneic hematopoietic cell transplantation in acute myeloid leukemia: A meta-analysis. Haematologica 102: 865-873, 2017.

32. Zeijlemaker W, Grob T, Meijer R, Hanekamp D, Kelder A, Carbaat-Ham JC, Oussoren-Brockhoff YJM, Snel AN, Veldhuizen D, Scholten WJ, et al: $\mathrm{CD} 34{ }^{+} \mathrm{CD} 38$-leukemic stem cell frequency to predict outcome in acute myeloid leukemia. Leukemia 33: 1102-1112, 2018.

33. Plesa A, Dumontet C, Mattei E, Tagoug I, Hayette S, Sujobert P, Tigaud I, Pages MP, Chelghoum Y, Baracco F, et al: High frequency of $\mathrm{CD} 34^{+} \mathrm{CD} 38^{\%} /$ low immature leukemia cells is correlated with unfavorable prognosis in acute myeloid leukemia. World J Stem Cells 9: 227-234, 2017.

34. Tomita H, Tanaka K, Tanaka T and Hara A: Aldehyde dehydrogenase 1A1 in stem cells and cancer. Oncotarget 7: 11018-11032, 2016.

35. Jaśkiewicz A, Pająk B and Orzechowski A: The many faces of Rap1 GTPase. Int J Mol Sci 19: pii: E2848, 2018.

36. Minato $\mathrm{N}$ and Hattori M: Spa-1 (Sipa1) and Rap signaling in leukemia and cancer metastasis. Cancer Sci 100: 17-23, 2009.

37. Rebhun JF, Castro AF and Quilliam LA: Identification of guanine nucleotide exchange factors (GEFs) for the Rap1 GTPase. Regulation of MR-GEF by M-Ras-GTP interaction. J Biol Chem 275: 34901-34908, 2000.

38. Sot B, Kötting C, Deaconescu D, Suveyzdis Y, Gerwert K and Wittinghofer A: Unravelling the mechanism of dual-specificity GAPs. EMBO J 29: 1205-1214, 2010.

This work is licensed under a Creative Commons Attribution-NonCommercial-NoDerivatives 4.0 International (CC BY-NC-ND 4.0) License. 